PROCEEDINGS OF THE

AMERICAN MATHEMATICAL SOCIETY

Volume 139, Number 1, January 2011, Pages 111-124

S 0002-9939(2010)10605-6

Article electronically published on July 30, 2010

\title{
ON FINITE ARITHMETIC SIMPLICIAL COMPLEXES
}

\author{
MIHRAN PAPIKIAN \\ (Communicated by Matthew A. Papanikolas)
}

\begin{abstract}
We compute the Euler-Poincaré characteristic of quotients of the Bruhat-Tits building of $\operatorname{PGL}(n)$ under the action of arithmetic groups arising from central division algebras over rational function fields of positive characteristic. We use this result to determine the structure of the quotient simplicial complex in certain cases.
\end{abstract}

\section{INTRODUCTION}

The purpose of this article is to generalize to higher dimensions the genus formula for modular curves of $\mathcal{D}$-elliptic sheaves proven in [12. The proof of this genus formula given in [12] relies in part on the arithmetic of $\mathcal{D}$-elliptic sheaves. In this paper we avoid the use of the theory of $\mathcal{D}$-elliptic sheaves, and work exclusively within the arithmetic of central division algebras over $F:=\mathbb{F}_{q}(T)$; here $\mathbb{F}_{q}$ denotes the finite field with $q$ elements and $T$ is an indeterminate.

Denote by $A=\mathbb{F}_{q}[T]$ the subring of $F$ formed by the polynomials in $T$. For $0 \neq f \in A$, let $\operatorname{deg}(f)$ be the degree of $f$ as a polynomial in $T$, and put $\operatorname{deg}(0)=$ $+\infty$. For $f / g \in F$ with $f, g \in A$, let $\operatorname{deg}(f / g):=\operatorname{deg}(f)-\operatorname{deg}(g)$. Then $-\operatorname{deg}$ defines a valuation on $F$; the corresponding place is denoted by $\infty$. Let $F_{\infty}$ be the completion of $F$ at $\infty$. Let $n \geq 2$ and let $D$ be a central division algebra over $F$ of dimension $n^{2}$. Assume $D \otimes_{F} F_{\infty}$ is isomorphic to the matrix algebra $\mathbb{M}_{n}\left(F_{\infty}\right)$. Fix a maximal $A$-order $\Lambda$ in $D$ and denote by $\Gamma:=\Lambda^{\times}$its group of units. Let $\mathcal{B}$ be the Bruhat-Tits building of $\operatorname{PGL}_{n}\left(F_{\infty}\right)$. The group $\Gamma$ acts on $\mathcal{B}$, and the quotient $\Gamma \backslash \mathcal{B}$ is a finite simplicial complex. The genus formula in [12] is equivalent to a formula for the Euler-Poincaré characteristic $\chi(\Gamma \backslash \mathcal{B})$ when $n=2$. In this paper we generalize this formula to an arbitrary prime $n$. We also determine the possible stabilizers in $\Gamma$ of simplices of $\mathcal{B}$, and the number of $\Gamma$-orbits of simplices with a given stabilizer (Theorem 3.13). These results are sufficient for determining $\Gamma \backslash \mathcal{B}$ in the case when $D$ is ramified at exactly two rational places (Theorem 3.19): in this special case, $\Gamma \backslash \mathcal{B}$ is sort of a "multi-layered" $(n-1)$-simplex. I am not aware of other instances where the quotient $\Gamma \backslash \mathcal{B}$ is explicitly determined when $n \geq 3$ (but see [16] for $\mathrm{GL}_{n}(A) \backslash \mathcal{B}$ ).

The quotients $\Gamma \backslash \mathcal{B}$ play an important role in many arithmetic problems, e.g. the theory of automorphic forms over function fields. For congruence subgroups $\Gamma$

Received by the editors March 24, 2010.

2010 Mathematics Subject Classification. Primary 11F06, 11G09; Secondary 20E08.

The author was supported in part by NSF grant DMS-0801208.

(C)2010 American Mathematical Society Reverts to public domain 28 years from publication 
of $\mathrm{GL}_{2}(A)$, the quotient graphs $\Gamma \backslash \mathcal{B}$ have been extensively studied by Gekeler and others in relation to the theory of Drinfeld modular forms; cf. [6], 7], 8].

The proof of Theorem 3.13 uses two key ingredients. One is Serre's theory relating Euler-Poincaré characteristics of discrete subgroups of non-Archimedean Lie groups to measures, and the other is Eichler's formula for the number of nonequivalent optimal embeddings of an order into a central simple algebra.

Notation. The following notation is fixed throughout the paper.

$$
\begin{aligned}
& |F|=\text { the set of places of } F . \\
& F_{x}=\text { the completion of } F \text { at } x \in|F| . \\
& \mathcal{O}_{x}=\left\{z \in F_{x} \mid \operatorname{ord}_{x}(z) \geq 0\right\}=\text { the ring of integers of } F_{x} . \\
& \pi_{x}=\left\{z \in F_{x} \mid \operatorname{ord}_{x}(z)>0\right\}=\text { the maximal ideal of } \mathcal{O}_{x} . \\
& \mathbb{F}_{x}=\mathcal{O}_{x} / \pi_{x} . \\
& q_{x}=\# \mathbb{F}_{x} . \\
& \operatorname{deg}(x)=\left[\mathbb{F}_{x}: \mathbb{F}_{q}\right] .
\end{aligned}
$$

Since the place $\infty$ plays a special role in our arguments, to simplify the notation we put $K:=F_{\infty}, \mathcal{O}:=\mathcal{O}_{\infty}, \pi:=\pi_{\infty}$, ord $:=\operatorname{ord}_{\infty}$.

\section{Bruhat-Tits Building of $\mathrm{PGL}_{n}(K)$}

2.1. Simplicial complexes. By a simplicial complex we mean a usual abstract simplicial complex, cf. [11, p. 15], except that we allow for two distinct simplices of the same positive dimension to have the same sets of vertices. More precisely, a simplicial complex $X$ is a collection of nonempty sets

$$
S_{0}(X), S_{1}(X), \ldots, S_{n}(X), \quad n \leq \infty,
$$

where each $s \in S_{i}(X)$ is a subset of $S_{0}(X)$ of cardinality $i+1$, and each subset of $s$ of cardinality $j+1,0 \leq j \leq i$, is in $S_{j}(X)$. We call $s \in S_{i}(X)$ an $i$-simplex and each nonempty subset of $s$ a face of $s$. The vertices of the simplex $s$ are the one-point elements of the subset $s \subset S_{0}(X)$. With this terminology, the elements of $S_{0}(X)$ are called the vertices of $X$. We will use the notation $\operatorname{Ver}(X)=S_{0}(X)$. If $n$ is finite, we call it the dimension of $X . X$ is finite if it is a finite set. The generalized $m$-th degree of $s \in X$, denoted $\operatorname{deg}_{X}^{m}(s)$, is the number of elements of $S_{m}(X)$ having $s$ as a face.

Let $X$ be finite of dimension $n$. The Euler-Poincaré characteristic of $X$ is

$$
\chi(X):=\sum_{i=0}^{n}(-1)^{i} \# S_{i}(X) .
$$

One can define the cohomology groups of $X$ (with $\mathbb{Q}$-coefficients) $H^{*}(X, \mathbb{Q})$ in the usual manner; cf. [11. Then

$$
\chi(X)=\sum_{i=0}^{n}(-1)^{i} \operatorname{dim}_{\mathbb{Q}} H^{i}(X, \mathbb{Q}) .
$$

We say that a group $G$ acts on $X$ if $G$ acts on the set of simplices of $X$ and this action satisfies the following condition: if $s \in S_{i}(X)$ has vertices $\left\{v_{0}, \ldots, v_{i}\right\}$, then $g s \in S_{i}(X)$ has vertices $\left\{g v_{0}, \ldots, g v_{i}\right\}, g \in G$. We single out an extra condition on the action of $G$ :

$$
\text { If } g s=s \text { for a simplex } s \text {, then } g \text { fixes all the vertices of } s \text {. }
$$


If $G$ acts on $X$ and satisfies (2.1), then there is a natural quotient simplicial complex $Y:=G \backslash X$ such that $S_{i}(Y)=S_{i}(X) / G$ for all $i$. For $s \in X$, denote by $O_{s}=G \cdot s$ the orbit of $s$ under the action of $G$, i.e., $O_{s}=\{g s \mid g \in G\}$. The action of $G$ decomposes $S_{i}(X)$ into a disjoint union of orbits $O_{s}$, and the set of these orbits is in bijection with $S_{i}(Y)$. Denote by

$$
G_{s}=\{g \in G \mid g s=s\}
$$

the stabilizer of $s$. Let $\tilde{s} \in Y$ and $s$ be a preimage of $\tilde{s}$ in $X$. We define $O_{\tilde{s}}=O_{s}$ and $\# G_{\tilde{s}}=\# G_{s}$; the second definition makes sense since the elements in the orbit $O_{s}$ have isomorphic stabilizers: $G_{g s}=g G_{s} g^{-1}$.

Lemma 2.1. Assume $G$ is finite and $X$ is finite of dimension $n$. With the previous notation,

$$
\chi(Y)=\frac{\chi(X)}{\# G}+\sum_{i=0}^{n}(-1)^{i} \sum_{\tilde{s} \in S_{i}(Y)}\left(1-\frac{1}{\# G_{\tilde{s}}}\right) .
$$

Proof. Since the orbits are disjoint, we have

$$
\begin{aligned}
\# S_{i}(X) & =\sum_{\tilde{s} \in S_{i}(Y)} \# O_{\tilde{s}}=\sum_{\tilde{s} \in S_{i}(Y)} \frac{\# G}{\# G_{\tilde{s}}} \\
& =\# G \# S_{i}(Y)+\sum_{\tilde{s} \in S_{i}(Y)} \# G\left(\frac{1}{\# G_{\tilde{s}}}-1\right) .
\end{aligned}
$$

Now take the alternating sums of both sides over $0 \leq i \leq n$.

2.2. The building. A lattice in $K^{n}$ is any finitely generated $\mathcal{O}$-submodule of $K^{n}$ which contains a basis of this vector space; such a module is free of rank $n$. If $x \in K^{\times}$and $L$ is a lattice in $K^{n}$, then $x L$ is also a lattice in $K^{n}$. Thus the group $K^{\times}$acts on the set of lattices $\mathcal{L}$. Denote the quotient $\mathcal{L} / K^{\times}$by $\overline{\mathcal{L}}$.

Define a simplicial complex $\mathcal{B}$ as follows. Let $\operatorname{Ver}(\mathcal{B})=\overline{\mathcal{L}}$. A finite subset of $\overline{\mathcal{L}}$ is an $i$-simplex of $\mathcal{B}$ if one can represent its elements by lattices $L_{0}, \ldots, L_{i}$ such that

$$
L_{0} \supsetneq L_{1} \supsetneq \cdots \supsetneq L_{i} \supsetneq \pi L_{0},
$$

and each simplex is uniquely determined by its vertices. The simplicial complex $\mathcal{B}$ is called the Bruhat-Tits building of $\mathrm{PGL}_{n}(K)$. Each $L_{i} / \pi L_{0}$ is a module over $\mathcal{O} / \pi \mathcal{O} \cong \mathbb{F}_{q}$, so from (2.2) we get a strictly decreasing chain of linear subspaces

$$
L_{0} / \pi L_{0} \supset L_{1} / \pi L_{0} \supset \cdots \supset L_{i} / \pi L_{0} \supset 0 .
$$

Since $L_{0} / \pi L_{0} \cong \mathbb{F}_{q}^{n}$ is $n$-dimensional over $\mathbb{F}_{q}, \mathcal{B}$ is an infinite $(n-1)$-dimensional simplicial complex. $\mathrm{GL}_{n}(K)$ acts on $\mathcal{B}$ via its natural action on the lattices (note that $\mathrm{GL}_{n}(K)$ preserves inclusions of lattices).

Definition 2.2. Let $L \in \mathcal{L}$ be spanned over $\mathcal{O}$ by the vectors $e_{1}, \ldots, e_{n}$ in $K^{n}$. Let $\operatorname{det}(L)$ be the determinant of the matrix having as its columns the elements $e_{1}, \ldots, e_{n}$. The type of $L$ is the element of $\mathbb{Z} / n \mathbb{Z}$ defined by

$$
\text { Type }(L):=\operatorname{ord}(\operatorname{det}(L)) \bmod n \text {. }
$$

Note that Type $(L)=\operatorname{Type}(x L)$ for any $x \in K^{\times}$, so we can associate types to the vertices of $\mathcal{B}$. It is easy to check that the vertices of any simplex in $\mathcal{B}$ have distinct types. The action of $\mathrm{GL}_{n}(K)$ on $\mathcal{B}$ does not preserve the types of vertices (in fact, $\mathrm{GL}_{n}(K)$ acts transitively on the vertices of $\left.\mathcal{B}\right)$. A matrix $g \in \mathrm{GL}_{n}(K)$ preserves the types of vertices if $n \mid \operatorname{ord}(\operatorname{det}(g))$. 
Notation 2.3. Let $z$ be a parameter. Set $[0]_{z}=1$. For $m \geq 1$, let

$$
[m]_{z}:=\left(z^{m}-1\right)\left(z^{m-1}-1\right) \cdots(z-1) .
$$

Definition 2.4. An ordered partition of $n$ is an expression of $n$ as an ordered sum of positive integers. We will write ordered partitions as row vectors:

$$
\mathbf{p}=\left(p_{1}, \ldots, p_{h}\right), \quad p_{1}, \ldots, p_{h} \geq 1, \quad n=p_{1}+\cdots+p_{h} .
$$

Define the length of $\mathbf{p}=\left(p_{1}, \ldots, p_{h}\right)$ to be $\ell(\mathbf{p}):=h$. The set of all ordered partitions of $n$ will be denoted by $\operatorname{Par}(n)$. For $\mathbf{p}=\left(p_{1}, \ldots, p_{h}\right) \in \operatorname{Par}(n)$, let

$$
\left[\begin{array}{l}
n \\
\mathbf{p}
\end{array}\right]_{q}:=\frac{[n]_{q}}{\left[p_{1}\right]_{q}\left[p_{2}\right]_{q} \cdots\left[p_{h}\right]_{q}} \text {. }
$$

It is obvious that $\left[\begin{array}{l}n \\ \mathbf{p}\end{array}\right]_{q}$ does not depend on the ordering of the entries of $\mathbf{p}$.

Lemma 2.5. Let $v \in \operatorname{Ver}(\mathcal{B})$. Then for $1 \leq i \leq n-1$,

$$
\operatorname{deg}_{\mathcal{B}}^{i}(v)=\sum_{\substack{\mathbf{p} \in \operatorname{Par}(n) \\
\ell(\mathbf{p})=i+1}}\left[\begin{array}{l}
n \\
\mathbf{p}
\end{array}\right]_{q} .
$$

Proof. Suppose $v$ corresponds to the class of the lattice $L$. Let $V:=L / \pi L \cong \mathbb{F}_{q}^{n}$. An $i$-flag in $V$ is a chain of vector subspaces

$$
V \neq \mathcal{F}_{1} \supsetneq \mathcal{F}_{2} \supsetneq \cdots \supsetneq \mathcal{F}_{i} \neq 0 .
$$

From the definition of $\mathcal{B}$ it is easy to see that the $i$-simplices of $\mathcal{B}$ having $v$ as a vertex are in bijection with the $i$-flags in $V$. Next, to each $i$-flag we associate an ordered partition of length $i+1$ as follows. Let $d_{i}:=\operatorname{dim}_{\mathbb{F}_{q}} \mathcal{F}_{i}$. Then to (2.3) we associate

$$
\left(n-d_{1}, d_{1}-d_{2}, \ldots, d_{i-1}-d_{i}, d_{i}\right) .
$$

Denote the number of $k$-dimensional subspaces in $\mathbb{F}_{q}^{m}$ by $\left[\begin{array}{c}m \\ k\end{array}\right]_{q}$. The number of distinct $i$-flags which map to $\mathbf{p}=\left(p_{1}, \ldots, p_{i+1}\right)$ is equal to

$$
f(\mathbf{p}):=\left[\begin{array}{c}
n \\
n-p_{1}
\end{array}\right]_{q}\left[\begin{array}{c}
n-p_{1} \\
n-p_{1}-p_{2}
\end{array}\right]_{q}\left[\begin{array}{c}
n-p_{1}-p_{2} \\
n-p_{1}-p_{2}-p_{3}
\end{array}\right]_{q} \ldots\left[\begin{array}{c}
n-p_{1}-\cdots-p_{i} \\
0
\end{array}\right]_{q} .
$$

It is well-known that $\left[\begin{array}{c}m \\ k\end{array}\right]_{q}=\frac{[m]_{q}}{[k]_{q}[m-k]_{q}}$, so $f(\mathbf{p})=\left[\begin{array}{l}n \\ \mathbf{p}\end{array}\right]_{q}$. Hence

$$
\operatorname{deg}_{\mathcal{B}}^{i}(v)=\sum_{\substack{\mathbf{p} \in \operatorname{Par}(n) \\
\ell(\mathbf{p})=i+1}} f(\mathbf{p})=\sum_{\substack{\mathbf{p} \in \operatorname{Par}(n) \\
\ell(\mathbf{p})=i+1}}\left[\begin{array}{l}
n \\
\mathbf{p}
\end{array}\right]_{q} .
$$

Example 2.6. Let $n=3$. The length- 2 ordered partitions of 3 are $(1,2)$ and $(2,1)$, so

$$
\operatorname{deg}_{\mathcal{B}}^{1}(v)=\left[\begin{array}{c}
3 \\
(1,2)
\end{array}\right]_{q}+\left[\begin{array}{c}
3 \\
(2,1)
\end{array}\right]_{q}=2 \frac{\left(q^{3}-1\right)\left(q^{2}-1\right)(q-1)}{\left(q^{2}-1\right)(q-1)(q-1)}=2\left(q^{2}+q+1\right) .
$$

Similarly,

$$
\operatorname{deg}_{\mathcal{B}}^{2}(v)=\left[\begin{array}{c}
3 \\
(1,1,1)
\end{array}\right]_{q}=\frac{\left(q^{3}-1\right)\left(q^{2}-1\right)(q-1)}{(q-1)^{3}}=\left(q^{2}+q+1\right)(q+1) .
$$


We will need the next lemma in 3 .

Lemma 2.7. For $n \geq 1$, we have

$$
\sum_{\mathbf{p} \in \operatorname{Par}(n)}(-1)^{\ell(\mathbf{p})} \frac{1}{\ell(\mathbf{p})}\left[\begin{array}{l}
n \\
\mathbf{p}
\end{array}\right]_{q}=(-1)^{n} \frac{1}{n}[n-1]_{q} .
$$

Proof. In this proof we treat $q$ as a formal parameter and manipulate infinite series and products ignoring the issues of convergence (for a justification, see [1]). We need to prove the following:

$$
\sum_{h=1}^{\infty} \frac{(-1)^{h}}{h} \sum_{\substack{p_{1}+\cdots+p_{h}=n \\ p_{1} \geq 1, \ldots, p_{h} \geq 1}} \frac{(-1)^{n}}{\left[p_{1}\right]_{q} \cdots\left[p_{h}\right]_{q}}=\frac{1}{n\left(q^{n}-1\right)} .
$$

We put the left-hand side into a generating series

$$
\begin{gathered}
\sum_{n=1}^{\infty} x^{n} \sum_{h=1}^{\infty} \frac{(-1)^{h}}{h} \sum_{\substack{p_{1}+\cdots+p_{h}=n \\
p_{1} \geq 1, \ldots, p_{h} \geq 1}} \frac{(-1)^{n}}{\left[p_{1}\right]_{q} \cdots\left[p_{h}\right]_{q}} \\
=\sum_{h=1}^{\infty} \frac{(-1)^{h}}{h} \sum_{p_{1} \geq 1, \ldots, p_{h} \geq 1} \frac{(-x)^{p_{1}+\cdots+p_{h}}}{\left[p_{1}\right]_{q} \cdots\left[p_{h}\right]_{q}} \\
=\sum_{h=1}^{\infty} \frac{(-1)^{h}}{h}\left(\sum_{m=1}^{\infty} \frac{(-x)^{m}}{[m]_{q}}\right)^{h}=-\ln (1+\mathfrak{E}),
\end{gathered}
$$

where $\mathfrak{E}:=\sum_{m=1}^{\infty}(-x)^{m} /[m]_{q}$. By a formula of Euler [1, Cor. 2.2],

$$
\mathfrak{E}=-1+\prod_{i=0}^{\infty}\left(1-x q^{i}\right)^{-1}
$$

so we have

$$
\begin{gathered}
-\ln (1+\mathfrak{E})=\sum_{i=0}^{\infty} \ln \left(1-x q^{i}\right)=-\sum_{i=0}^{\infty} \sum_{n=1}^{\infty} \frac{\left(x q^{i}\right)^{n}}{n} \\
=-\sum_{n=1}^{\infty} \frac{x^{n}}{n} \sum_{i=0}^{\infty} q^{i n}=\sum_{n=1}^{\infty} \frac{x^{n}}{n\left(q^{n}-1\right)} .
\end{gathered}
$$

We conclude that the coefficient of $x^{n}$ in the initial generating series is equal to $1 / n\left(q^{n}-1\right)$, which finishes the proof.

2.3. Euler-Poincaré measure. Denote $G:=\mathrm{PGL}_{n}(K) . G$ is a locally compact unimodular topological group. Let $d g$ be the Haar measure on $\mathrm{GL}_{n}(K)$ normalized by $\operatorname{Vol}\left(\mathrm{GL}_{n}(\mathcal{O}), d g\right)=1$. Let $d z$ be the Haar measure on $K^{\times}$normalized by $\operatorname{Vol}\left(\mathcal{O}^{\times}, d z\right)=1$. Let $d h:=d g / d z$ be the quotient measure on $G=\mathrm{GL}_{n}(K) / K^{\times}$. Let $\Gamma$ be a discrete subgroup of $G$ and assume that $\Gamma \backslash G$ is compact. Since $G$ acts on $\mathcal{B}$ via its natural action on lattices in $\overline{\mathcal{L}}, \Gamma$ also acts on $\mathcal{B}$. Assume that $\Gamma$ preserves the types of vertices of $\mathcal{B}$. Then $\Gamma$ satisfies $(2.1)$, since the types of vertices of any simplex are distinct. The quotient simplicial complex $\Gamma \backslash \mathcal{B}$ is finite since $\Gamma \backslash G$ is compact; cf. [15, p. 139]. Let $d \delta$ be the counting measure on $\Gamma$, and $d h / d \delta$ be the quotient measure on $\Gamma \backslash G$. The stabilizer $\Gamma_{t}$ of $t \in \mathcal{B}$ is finite since the stabilizer of $t$ in $G$ is bounded and $\Gamma$ is discrete in $G$; cf. [15, p. 115]. The order 
$\# \Gamma_{s}$ does not depend on the choice of $s$ in the orbit $\Gamma \cdot s$. Therefore, for $s \in \Gamma \backslash \mathcal{B}$, we can define $\# \Gamma_{s}$ as $\# \Gamma_{t}$ for some preimage $t$ of $s$ in $\mathcal{B}$.

Theorem 2.8. Assume $\Gamma$ has a normal torsion-free subgroup of finite index. Then

$$
\begin{aligned}
\chi(\Gamma \backslash \mathcal{B})= & \frac{1}{n}(-1)^{n-1}[n-1]_{q} \operatorname{Vol}\left(\Gamma \backslash G, \frac{d h}{d \delta}\right) \\
& +\sum_{i=0}^{n-1}(-1)^{i} \sum_{s \in S_{i}(\Gamma \backslash \mathcal{B})}\left(1-\frac{1}{\# \Gamma_{s}}\right) .
\end{aligned}
$$

Proof. $G$ has an Euler-Poincaré measure $\mu$ in the sense of [15] (see page 140 in [15]); in fact, $\mu$ is necessarily unique. Let $\Gamma^{\prime} \triangleleft \Gamma$ be a normal torsion-free subgroup of finite index. Then $\Gamma^{\prime}$ is a cocompact subgroup of $G$, and hence is of type (FL) in the terminology of [15] (see Theorem 3 on page 121 of [15]). Therefore, by the definition of $\mu, \chi\left(\Gamma^{\prime}\right)=\operatorname{Vol}\left(\Gamma^{\prime} \backslash G, \mu / d \delta\right)$. Since the geometric realization of $\mathcal{B}$ is contractible, $\chi\left(\Gamma^{\prime}\right)=\chi\left(\Gamma^{\prime} \backslash \mathcal{B}\right)$ (see Proposition 9 on page 91 in [15]) We conclude that $\chi\left(\Gamma^{\prime} \backslash \mathcal{B}\right)=\operatorname{Vol}\left(\Gamma^{\prime} \backslash G, \mu / d \delta\right)$. Since $\mu$ is a Haar measure, it is proportional to any other Haar measure $\mu^{\prime}$ on $G$, i.e., $\mu=c \cdot \mu^{\prime}$ for some nonzero constant $c \in \mathbb{R}$. A method for computing this constant is given on page 140 of [15]. For the measure $d h$, Theorem 7 on page 150 of [15] gives

$$
\mu=\frac{1}{n}(-1)^{n-1}[n-1]_{q} d h ;
$$

see also [10, Prop 5.3.9]. Thus,

$$
\chi\left(\Gamma^{\prime} \backslash \mathcal{B}\right)=\frac{1}{n}(-1)^{n-1}[n-1]_{q} \operatorname{Vol}\left(\Gamma^{\prime} \backslash G, \frac{d h}{d \delta}\right) .
$$

Let $H:=\Gamma^{\prime} \backslash \Gamma$. Then $H$ acts on $\Gamma^{\prime} \backslash \mathcal{B}$ and $\Gamma \backslash \mathcal{B}=H \backslash\left(\Gamma^{\prime} \backslash \mathcal{B}\right)$. Let $s \in S_{i}(\Gamma \backslash \mathcal{B})$. Since $\Gamma^{\prime}$ is torsion-free, it is easy to see that $\# H_{s}=\# \Gamma_{s}$. Hence by Lemma 2.1

$$
\begin{aligned}
\chi(\Gamma \backslash \mathcal{B})= & \frac{1}{n}(-1)^{n-1}[n-1]_{q} \frac{1}{\# H} \operatorname{Vol}\left(\Gamma^{\prime} \backslash G, \frac{d h}{d \delta}\right) \\
& +\sum_{i=0}^{n-1}(-1)^{i} \sum_{s \in S_{i}(\Gamma \backslash \mathcal{B})}\left(1-\frac{1}{\# \Gamma_{s}}\right) .
\end{aligned}
$$

Finally, $\frac{1}{\# H} \operatorname{Vol}\left(\Gamma^{\prime} \backslash G, \frac{d h}{d \delta}\right)=\operatorname{Vol}\left(\Gamma \backslash G, \frac{d h}{d \delta}\right)$.

\section{Quotients By ARIThmetic GRoups}

Let $D$ be a central division algebra over $F$ of dimension $n^{2}$. For $x \in|F|$, let $D_{x}:=D \otimes_{F} F_{x}$ and $\operatorname{inv}_{x}(D) \in \mathbb{Q} / \mathbb{Z}$ be the local invariant of $D$ at $x ;$ see $[14$, Ch. 8] for the definition. Let $R \subset|F|$ be the set of places ramified in $D$, i.e., the set of places for which $\operatorname{inv}_{x}(D) \neq 0$. The following facts can be found in [14, §32]:

(1) For any $x \in|F|$ there exists $m_{x} \mid n$ such that $m_{x} \cdot \operatorname{inv}_{x}(D)=0$.

(2) $n$ is the smallest positive integer such that $n \cdot \operatorname{inv}_{x}(D)=0$ for all $x$.

(3) $R$ is a finite set and $\sum_{x \in R} \operatorname{inv}_{x}(D)=0$.

(4) $D$ is uniquely determined by its local invariants.

These properties obviously imply that $\# R \geq 2$. Assume $D_{x}$ is a division algebra over $F_{x}$ for each $x \in R$; this is equivalent to $\operatorname{inv}_{x}(D)$ having exponent $n$ in $\mathbb{Q} / \mathbb{Z}$. (Later in the section we will assume that $n$ is prime, which makes this condition 
automatic.) Note that this assumption, combined with (3), implies that \#R is even for even $n$. From now on we assume $\infty \notin R$.

Let $\Lambda$ be a maximal $A$-order in $D$. Since $D$ satisfies the Eichler condition [14, (34.3)] and $A$ is a principal ideal domain, $\Lambda$ is unique up to conjugation in $D$; see [14, (35.14)]. Let $\Gamma:=\Lambda^{\times}$be the subgroup of units of $\Lambda$. This is the subset of $\Lambda$ consisting of those elements whose reduced norm is in $\mathbb{F}_{q}^{\times}$. Let $D^{\times}$be the multiplicative group of $D . \Gamma$ acts on $\mathcal{B}$ via the embedding

$$
\Gamma \hookrightarrow D^{\times} \hookrightarrow(D \otimes K)^{\times} \cong \mathrm{GL}_{n}(K) .
$$

Let $\bar{\Gamma}$ be the image of $\Gamma$ in $G:=\operatorname{PGL}_{n}(K)$. Note that $\mathbb{F}_{q}^{\times}$is in the center of $\Gamma$ and $\bar{\Gamma} \cong \Gamma / \mathbb{F}_{q}^{\times} . \bar{\Gamma}$ is a discrete cocompact subgroup of $G$. Moreover, $\Gamma$ preserves the types of vertices of $\mathcal{B}$ since $\operatorname{ord}(\operatorname{det}(\gamma))=0$ for any $\gamma \in \Gamma$. Hence we can apply Theorem 2.8 to compute the Euler-Poincaré characteristic of $\Gamma \backslash \mathcal{B}=\bar{\Gamma} \backslash \mathcal{B}\left(\mathbb{F}_{q}^{\times}\right.$acts trivially on $\mathcal{B}$ ), but first we need to determine the stabilizers of simplices in $\Gamma \backslash \mathcal{B}$. This will be done in a series of lemmas. For $x \in|F|-\infty$, denote $\Lambda_{x}=\Lambda \otimes_{A} \mathcal{O}_{x}$.

Lemma 3.1. Let $H$ be a finite subgroup of $\Gamma$. Then there exists a vertex $v \in \operatorname{Ver}(\mathcal{B})$ such that $H \subset \Gamma_{v}$.

Proof. This is a consequence of the Bruhat-Tits fixed point theorem; see the theorem on page 161 in [2].

Lemma 3.2. Let $H$ be a finite subgroup of $\Gamma$. Then $H$ is isomorphic to a subgroup of $\mathrm{GL}_{n}\left(\mathbb{F}_{q}\right)$ of order coprime to $p$.

Proof. First, we show that every element of $H$ has order coprime to $p$. Let $g \in H$ be of order $m$. Suppose $p \mid m$. Replacing $g$ by $g^{m / p}$, we may assume that $g$ has order $p$. Now $g^{p}=1$ implies that $(g-1)^{p}=0$ in $D$. Since $g \neq 1$, this leads to a contradiction, as $D$ is a division algebra. By Cauchy's theorem, $p$ is coprime to $\# H$. Next, by Lemma 3.1, we know that $H \subset \Gamma_{v}$ for some vertex $v \in \mathcal{B}$. The stabilizer of $v$ in $\mathrm{GL}_{n}(K)$ is isomorphic to $K^{\times} \mathrm{GL}_{n}(\mathcal{O})$. Hence $H$ is isomorphic to a subgroup of $\mathrm{GL}_{n}(\mathcal{O})$. Consider the reduction map $\mathrm{GL}_{n}(\mathcal{O}) \rightarrow \mathrm{GL}_{n}\left(\mathbb{F}_{q}\right)$. It is well known that the kernel of this homomorphism contains torsion elements only of order a power of $p$, so $H$ maps isomorphically to a subgroup of $\mathrm{GL}_{n}\left(\mathbb{F}_{q}\right)$.

\section{Lemma 3.3.}

(1) Every finite subgroup of $\Gamma$ is contained in a maximal finite subgroup.

(2) A maximal finite subgroup of $\Gamma$ is isomorphic to $\mathbb{F}_{q^{d}}^{\times}$for some $d \mid n$. Moreover, if $D^{\times}$contains a subgroup isomorphic to $\mathbb{F}_{q^{n}}^{\times}$, then every maximal finite subgroup of $\Gamma$ is isomorphic to $\mathbb{F}_{q^{n}}^{\times}$.

(3) The stabilizer in $\Gamma$ of a simplex of $\mathcal{B}$ is isomorphic to $\mathbb{F}_{q^{d}}^{\times}$for some $d \mid n$.

Proof. (1) Each sequence of finite subgroups of $\Gamma$ ordered by inclusion contains a maximal element since all such subgroups are isomorphic to subgroups of $\mathrm{GL}_{n}\left(\mathbb{F}_{q}\right)$.

(2) Let $H$ be a maximal finite subgroup of $\Gamma$. We can consider $H$ as a finite subgroup of $\Lambda_{x}^{\times}, x \in|F|-\infty$. In particular, let $x \in R$. Then by assumption $D_{x}$ is a central division algebra over $F_{x}$, so $\Lambda_{x}$ is the unique $\mathcal{O}_{x}$-maximal order in $D_{x}$. The structure of $\Lambda_{x}$ is well-known: $\Lambda_{x}$ has a unique two-sided maximal ideal $\Pi_{x}$ and $\Lambda_{x} / \Pi_{x} \cong \mathbb{F}_{x}^{(n)}$, where $\mathbb{F}_{x}^{(n)}$ denotes the degree- $n$ extension of $\mathbb{F}_{x}$; see [14, Ch. 3]. Hence a finite subgroup of $\Lambda_{x}^{\times}$is isomorphic to a subgroup of $\left(\mathbb{F}_{x}^{(n)}\right)^{\times}$. In particular, $H$ is commutative. Since $H$ is finite and commutative, the subring $\mathbb{F}_{q}[H]$ 
of $\Lambda$ generated over $\mathbb{F}_{q}$ by $H$ is finite. On the other hand, $D$ is a division algebra. Hence $\mathbb{F}_{q}[H]$ is a finite field extension $\mathbb{F}$ of $\mathbb{F}_{q}$. Obviously, $\mathbb{F}^{\times} \subset \Lambda^{\times}=\Gamma$; thus $H=\mathbb{F}^{\times}$by maximality. Now $L:=\mathbb{F} F$ is an $F$-subfield of $D$ and $[L: F]=\left[\mathbb{F}: \mathbb{F}_{q}\right]$. This implies that $d:=\left[\mathbb{F}_{:}: \mathbb{F}_{q}\right]$ divides $n$ (see [10, Cor. A.3.4, p. 255]), and $H \cong \mathbb{F}_{q^{d}}^{\times}$.

Let $L^{\prime}:=\mathbb{F}_{q^{n}} F$. Assume $L^{\prime}$ embeds into $D$. Any two embeddings of $L$ into $D$ are conjugate; cf. [10, Cor. A.3.4, p. 255]. Hence any subfield of $D$ isomorphic to $L$ is contained in a field isomorphic to $L^{\prime}$, and $L \cap \Lambda \subset L^{\prime} \cap \Lambda$. Suppose $\tau \in L^{\prime}$ generates $\mathbb{F}_{q^{n}}^{\times}$. Then some power of $\tau$ generates $H$, so $\tau \in \Gamma$. This implies that $H$ is contained in a subgroup in $\Gamma$ isomorphic to $\mathbb{F}_{q^{n}}^{\times}$. By maximality, $H \cong \mathbb{F}_{q^{n}}^{\times}$.

(3) The proof of this part is similar to (2). Let $s$ be a simplex of $\mathcal{B}$ and $H=\Gamma_{s}$. The stabilizer of $s$ in $\mathrm{GL}_{n}(K)$ is $K^{\times} \cdot P$ for some parahoric subgroup $P$, so $H=\Gamma \cap P$; see [2, VI.5]. Let $\mathbb{F}^{\times}:=\mathbb{F}_{q}[H]^{\times}$. As we saw in (2), $\mathbb{F}$ is a field and $\mathbb{F}^{\times} \cong \mathbb{F}_{q^{d}}$ for some $d \mid n$. The lattices in $K^{n}$ forming the flag corresponding to $s$ are clearly mapped to themselves under the action of $\mathbb{F}$ (as a subring of $\mathbb{M}_{n}(K)$ ). Since the corresponding elements are invertible, they lie in $P$. Hence $H \subset \mathbb{F}^{\times} \subset \Gamma \cap P=H$. This implies that $\mathbb{F}^{\times}=H$.

Lemma 3.4. Let $H \subset \Gamma$ be a finite subgroup isomorphic to $\mathbb{F}_{q^{n}}^{\times}$. Then $H$ fixes a unique vertex of $\mathcal{B}$.

Proof. By Lemma 3.1, $H$ fixes a vertex $v$ in $\mathcal{B}$. Assume there is another vertex $w \neq v$ fixed by $H$. Let $B$ be the Euclidean building associated to $\mathcal{B}$; see [2, Ch. VI] for the definition. Let $[v, w]$ be the line segment in $B$ joining $v$ and $w$; this is welldefined by part (4) of the theorem on page 152 in [2. $H$ fixes $[v, w]$ pointwise since this is a group of isometries fixing the endpoints. The union of closed simplices containing $v$ is a neighborhood of $v$ in $B$. Therefore, there is a unique simplex $s \in \mathcal{B}$ of minimal positive dimension which contains $v$ and intersects $[v, w]$. This simplex must be fixed by $H$. This implies that $H$ is contained in a proper parahoric subgroup of $\mathrm{GL}_{n}(K)$; cf. the proof of Lemma 3.3. After conjugation, we can assume that $H$ is contained in a standard nonmaximal parahoric subgroup $P$ of $\mathrm{GL}_{n}(\mathcal{O})$. Let $\xi$ be a generator of $\mathbb{F}_{q^{n}}^{\times}$. Consider $\xi$ as a matrix in $\operatorname{GL}_{n}(\mathcal{O})$ and let $f_{\xi}$ be its characteristic polynomial. Then $f_{\xi}$ coincides with the minimal polynomial of $\xi$ over $\mathbb{F}_{q}$, so it is irreducible. The image of $\xi$ in $\mathrm{GL}_{n}\left(\mathbb{F}_{\infty}\right)$ under the reduction map $\mathrm{GL}_{n}(\mathcal{O}) \rightarrow \mathrm{GL}_{n}\left(\mathbb{F}_{\infty}\right)$ has characteristic polynomial $f_{\xi}\left(\bmod \pi_{\infty}\right)=f_{\xi}$, which is still irreducible since $\mathbb{F}_{\infty} \cong \mathbb{F}_{q}$. On the other hand, the image of $P$ in $\mathrm{GL}_{n}\left(\mathbb{F}_{\infty}\right)$ is a proper parabolic subgroup, so the characteristic polynomials of its elements are reducible. This leads to a contradiction.

Notation 3.5. Let $x \in|F|$ and let $m \geq 1$ be a positive integer. Denote

$$
\wp(x, m)= \begin{cases}0, & \text { if } \operatorname{gcd}(m, \operatorname{deg}(x))>1 ; \\ 1, & \text { otherwise. }\end{cases}
$$

Let $\wp(R, m):=\prod_{x \in R} \wp(x, m)$.

Lemma 3.6. $\mathbb{F}_{q^{m}}(T)$ embeds into $D$ if and only if $m \mid n$ and $\wp(R, m)=1$.

Proof. Let $K / F$ be a finite field extension. Then $K$ embeds into $D$ as an $F$ subalgebra if and only if $[K: F]$ divides $n$ and none of the places in $R$ split in $K$; see [10, Cor. A.3.4, p. 255]. There are no ramified places in the extension $\mathbb{F}_{q^{m}} F / F$. Moreover, a place $x \in|F|$ splits into $\operatorname{gcd}(\operatorname{deg}(x), m)$ places in $\mathbb{F}_{q^{m}}(T)$. 
Definition 3.7. Let $L:=\mathbb{F}_{q^{n}}(T)$ and $B:=\mathbb{F}_{q^{n}}[T] ; B$ is the integral closure of $A$ in $L$. Suppose there exists an embedding $\phi: L \hookrightarrow D$. We say that $\phi$ is an optimal embedding with respect to $\Lambda / B$ if $\phi(L) \cap \Lambda=\phi(B)$; cf. [17, p. 26]. For simplicity, we say that $B$ is optimally embedded in $\Lambda$. If $\phi$ exists, then it is known that $B$ is optimally embedded in at least one maximal $A$-order in $D$; cf. [4, p. 384]. Since all such maximal $A$-orders are conjugate in $D$, we can assume that $B$ is optimally embedded in $\Lambda$. Two optimal embeddings $\phi_{1}$ and $\phi_{2}$ of $B$ into $\Lambda$ are said to be equivalent modulo $\Gamma$ if $\phi_{2}=\gamma \phi_{1} \gamma^{-1}$ for some $\gamma \in \Gamma$. Denote the number of optimal embeddings of $B$ into $\Lambda$, which are nonequivalent modulo $\Gamma$, by $m(B)$. Similarly, for $x \in|F|-\infty$, denote by $m_{x}\left(B_{x}\right)$ the number of optimal embeddings of $B_{x}:=B \otimes_{A} \mathcal{O}_{x}$ into $\Lambda_{x}$ which are not equivalent modulo $\Lambda_{x}^{\times}$.

Theorem 3.8 (Eichler).

$$
m(B)=\prod_{x \in|F|-\infty} m_{x}\left(B_{x}\right) .
$$

Proof. The idelic proof of this fact given in [17, Thm. 5.11, p. 92] in the case of quaternion algebras extends directly to our case.

Lemma 3.9. If $x \notin R$, then $m_{x}\left(B_{x}\right)=1$.

Proof. To prove the lemma it is enough to show that there is a unique optimal embedding of $B$ into $\mathbb{M}_{n}(A)$, up to conjugation by $\mathrm{GL}_{n}(A)$. Indeed, if $x \notin R$, then $D_{x} \cong \mathbb{M}_{n}\left(F_{x}\right)$ and $\Lambda_{x}^{\times} \cong \mathrm{GL}_{n}\left(\mathcal{O}_{x}\right)$. We can apply Theorem 3.8 to $\mathbb{M}_{n}(F)$ with $\Lambda=\mathbb{M}_{n}(A)$. If we show that there is a unique equivalence class of optimal embeddings of $B$ into $\mathbb{M}_{n}(A)$, then $m(B)=1$, so by Eichler's theorem, $m_{x}\left(B_{x}\right)=1$.

Fix a generator $\xi$ of $\mathbb{F}_{q^{n}}$ over $\mathbb{F}_{q}$, i.e., $\mathbb{F}_{q^{n}}=\mathbb{F}_{q}[\xi]$. The minimal polynomial $f_{\xi}$ of $\xi$ over $\mathbb{F}_{q}$ has degree $n: f_{\xi}(x)=x^{n}+a_{n-1} x^{n-1}+\cdots+a_{n}$. Note that $B=A[\xi]$. To give an optimal embedding of $B$ into $\mathbb{M}_{n}(A)$ is equivalent to specifying a matrix $X$ in $\mathrm{GL}_{n}(A)$ whose minimal polynomial is $f_{\xi}$. The subring of $\mathbb{M}_{n}(A)$ generated by $X$ and the scalar matrices is isomorphic to $B$. The claim becomes the following statement. There exists a matrix in $\mathrm{GL}_{n}(A)$ with minimal polynomial $f_{\xi}$ and any two such matrices are conjugate in $\mathrm{GL}_{n}(A)$.

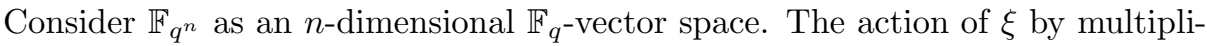
cation induces a linear transformation whose minimal polynomial is $f_{\xi}$. This proves the existence of the desired matrix $X$, since $\mathrm{GL}_{n}\left(\mathbb{F}_{q}\right) \subset \mathrm{GL}_{n}(A)$. The uniqueness of the conjugacy class of $X$ in $\mathrm{GL}_{n}(A)$ follows from the main theorem of $[9$, since $B$ is a principal ideal domain.

Lemma 3.10. If $x \in R$, then $m_{x}\left(B_{x}\right)=\wp(x, n) \cdot n$.

Proof. If $x \in R$, then $D_{x}$ is a division algebra by assumption. If $\wp(x, n)=0$, then $L_{x}:=L \otimes_{F} F_{x}$ is not a field, hence cannot be embedded into $D_{x}$. Now assume $\wp(x, n)=1$, so that there exists an embedding $L_{x} \hookrightarrow D_{x}$. Let $\operatorname{inv}_{x}(D)=d / n$ and let $\xi$ be as in the proof of Lemma 3.9. Then

$$
\Lambda_{x}=B_{x} \oplus B_{x} \tau \oplus \cdots \oplus B_{x} \tau^{n-1},
$$

where $\tau^{n}=\varpi_{x}^{d}$ (here $\varpi_{x}$ is a fixed uniformizer of $\mathcal{O}_{x}$ ), $\tau a=a \tau$ for $a \in \mathcal{O}_{x}$ and $\tau \xi=\xi^{q} \tau$; see (A.2.6) on page 253 in 10. The element $\tau$ generates a two-sided ideal $(\tau)$. Any element in this ideal has reduced norm divisible by $\pi_{x}$, so cannot be invertible. We conclude that $\Lambda_{x}^{\times}=B_{x}^{\times}$. To give an optimal embedding of $B_{x}$ into 
$\Lambda_{x}$ is equivalent to specifying an element of $\Lambda_{x}$ with minimal polynomial $f_{\xi}$. But these elements in $\Lambda_{x}$ are exactly $\xi, \xi^{q}, \ldots, \xi^{q^{n-1}}$. These elements are conjugate in $\Lambda_{x}$ only by elements in $(\tau)$, which is not in $\Lambda_{x}^{\times}$. Hence there are $n$ distinct optimal embeddings.

Proposition 3.11. The number of conjugacy classes of subgroups of $\Gamma$ isomorphic to $\mathbb{F}_{q^{n}}^{\times}$is equal to $\wp(R, n) n^{\# R-1}$.

Proof. We keep the notation of the proof of Lemma 3.9. Giving an optimal embedding of $B$ into $\Lambda$ is equivalent to specifying an element in $\Lambda$ with minimal polynomial $f_{\xi}$. Hence the number of optimal embeddings of $B$ into $\Lambda$ up to conjugation by $\Gamma$ is equal to the number of conjugacy classes of elements in $\Gamma$ with minimal polynomial $f_{\xi}$. By Theorem 3.8 and Lemmas 3.9, 3.10 the number of such conjugacy classes is equal to $\wp(R, n) n^{\# R}$. The cyclic subgroup generated in $\Gamma$ by an element with minimal polynomial $f_{\xi}$ is finite and isomorphic to $\mathbb{F}_{q^{n}}^{\times}$. Conversely, in a subgroup of $\Gamma$ isomorphic to $\mathbb{F}_{q^{n}}^{\times}$we can find exactly $n$ elements with minimal polynomial $f_{\xi}$ (over $F$ ). Hence to prove the proposition it remains to show that if $\gamma_{1} \neq \gamma_{2}$ satisfy $f_{\xi}\left(\gamma_{i}\right)=0$ and generate the same subgroup in $\Gamma$, then they are not $\Gamma$-conjugate. Suppose $\gamma_{2}=\gamma \gamma_{1} \gamma^{-1}$ with $\gamma \in \Gamma$. Then $\gamma_{1}$ and $\gamma_{2}$ are also conjugate in $\Lambda_{x}^{\times}$for $x \in R$. But as we saw in the proof of Lemma 3.10 this is not the case.

Proposition 3.12. The number of $\Gamma$-orbits of vertices of $\mathcal{B}$ with stabilizers isomorphic to $\mathbb{F}_{q^{n}}^{\times}$is equal to $\wp(R, n) n^{\# R-1}$.

Proof. Using Proposition 3.11 it is enough to show that there is a one-to-one correspondence between the set $S$ of $\Gamma$-orbits in $\operatorname{Ver}(\mathcal{B})$ with stabilizers isomorphic to $\mathbb{F}_{q^{n}}^{\times}$and the set $S^{\prime}$ of conjugacy classes of subgroups of $\Gamma$ isomorphic to $\mathbb{F}_{q^{n}}^{\times}$.

Since the vertices in the same $\Gamma$-orbit have $\Gamma$-conjugate stabilizers, the map $S \rightarrow S^{\prime}$ given by $v \mapsto \Gamma_{v}$ is well-defined. This map is surjective by Lemma 3.1. If the map is not injective, then there exist $v$ and $w$, which are not in the same $\Gamma$-orbit but $\Gamma_{w}=\gamma \Gamma_{v} \gamma^{-1}$. Now $\gamma v \neq w$, but their stabilizers are equal in $\Gamma$. In particular a subgroup of $\Gamma$ isomorphic to $\mathbb{F}_{q^{n}}^{\times}$fixes two distinct vertices in $\mathcal{B}$, which contradicts Lemma 3.4 .

Theorem 3.13. Assume $n$ is prime.

(1) If $s \in S_{i}(\mathcal{B})$ with $i \geq 1$, then $\Gamma_{s}=\mathbb{F}_{q}^{\times}$.

(2) For a vertex $v \in \operatorname{Ver}(\mathcal{B})$, the stabilizer $\Gamma_{v}$ is either $\mathbb{F}_{q}^{\times}$or is isomorphic to $\mathbb{F}_{q^{n}}^{\times}$. The number of $\Gamma$-orbits of vertices of $\mathcal{B}$ with $\Gamma_{v} \cong \mathbb{F}_{q^{n}}^{\times}$is equal to $\wp(R, n) n^{\# R-1}$.

$$
\chi(\Gamma \backslash \mathcal{B})=\frac{(q-1)(-1)^{n-1}}{[n]_{q}} \prod_{x \in R}[n-1]_{q_{x}}+\wp(R, n) \cdot n^{\# R-1}\left(1-\frac{q-1}{q^{n}-1}\right) .
$$

Proof. Let $s$ be an $i$-simplex of $\mathcal{B}$. By Lemma 3.3, $\Gamma_{s} \cong \mathbb{F}_{q^{d}}^{\times}$for some $d \mid n$. If $n$ is prime, then $d=1$ or $d=n$. Suppose $i>0$. Since $\Gamma_{s}$ fixes all the vertices of $s, \Gamma_{s}$ fixes at least two distinct vertices of $\mathcal{B}$. By Lemma $3.4 \Gamma_{s} \neq \mathbb{F}_{q^{n}}^{\times}$, so $\Gamma_{s}=\mathbb{F}_{q}^{\times}$. On the other hand, by Proposition 3.12, the number of $\Gamma$-orbits of vertices of $\mathcal{B}$ with stabilizers isomorphic to $\mathbb{F}_{q^{n}}^{\times}$is equal to $\wp(R, n) n^{\# R-1}$. This proves (1) and (2). 
By Proposition 4.1 and (6.9) in [13,

$$
\begin{aligned}
\operatorname{Vol}\left(\bar{\Gamma} \backslash G, \frac{d h}{d \delta}\right) & =n \frac{(q-1)(-1)^{\# R \cdot(n-1)}}{[n-1]_{q}[n]_{q}} \prod_{x \in R}[n-1]_{q_{x}} \\
& =\frac{n(q-1)}{[n-1]_{q}[n]_{q}} \prod_{x \in R}[n-1]_{q_{x}} .
\end{aligned}
$$

(The last equality follows from the fact that $\# R \cdot(n-1)$ is even for $n$ a prime.)

$\bar{\Gamma}$ has normal torsion-free subgroups of finite index; for example, the image in $G$ of a principal congruence subgroup of $\Gamma$ is such a subgroup. Now the formula of (3) follows from Theorem 2.8 .

Corollary 3.14. If $n$ is prime, then $\chi(\Gamma \backslash \mathcal{B}) \equiv 1(\bmod q)$.

Proof. From the formula for $\chi(\Gamma \backslash \mathcal{B})$ in Theorem 3.13 , it is easy to see that $\chi(\Gamma \backslash \mathcal{B}) \equiv$

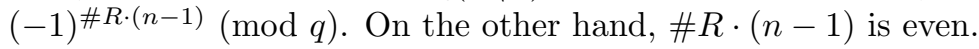

Example 3.15. Let $n=3$ and $R=\{x, y\}$ with $\operatorname{deg}(x)=\operatorname{deg}(y)=1$. Then

$$
\chi(\Gamma \backslash \mathcal{B})=\frac{(q-1)^{3}\left(q^{2}-1\right)^{2}}{(q-1)\left(q^{2}-1\right)\left(q^{3}-1\right)}+3\left(1-\frac{q-1}{q^{3}-1}\right)=q+1 .
$$

Let $n=3$ and $R=\{x, y\}$ with $\operatorname{deg}(x)=1$ and $\operatorname{deg}(y)=3$. Then

$$
\chi(\Gamma \backslash \mathcal{B})=\frac{(q-1)^{2}\left(q^{2}-1\right)\left(q^{3}-1\right)\left(q^{6}-1\right)}{(q-1)\left(q^{2}-1\right)\left(q^{3}-1\right)}=(q-1)\left(q^{6}-1\right) .
$$

Remark 3.16. The first equality in (3.1) is proven in [13] for arbitrary $n$, assuming $D_{x}$ is a division algebra for all $x \in R$. Note that this assumption also implies the second equality in (3.1) as then \#R $(n-1)$ is even (see the beginning of this section). If $\wp(R, m)=0$ for every $m>1$ dividing $n$, then by Lemma 3.6 the only torsion elements in $\Gamma$ are the elements of the center $\mathbb{F}_{q}^{\times}$. Now one can apply Theorem 2.8 to conclude that $\chi(\Gamma \backslash \mathcal{B})$ is given by the formula in Theorem 3.13 On the other hand, if we do not assume $\wp(R, m)=0$ for every $m>1$ dividing $n$, then the assumption on $n$ being prime cannot be omitted from Theorem 3.13. For example, if we take $n=4$ and $R=\{x, y\}$ with $\operatorname{deg}(x)=\operatorname{deg}(y)=1$, then the formula for $\chi(\Gamma \backslash \mathcal{B})$ in Theorem 3.13 generally gives noninteger values.

Remark 3.17. It is not completely obvious that the formula for $\chi(\Gamma \backslash \mathcal{B})$ in Theorem 3.13 always produces integer values. As we indicated in the previous remark, the requirement on $n$ being prime is necessary to make this happen. To see that the formula produces integer values, one can argue as follows:

First, suppose $n$ divides $\operatorname{deg}(y)$ for some $y \in R$, i.e., $\wp(R, n)=0$. Fix some $z \in R, z \neq y$. We have $[n]_{q}=\left(q^{n}-1\right)[n-1]_{q}$. Now $\left(q^{n}-1\right)$ divides $\left(q_{y}-1\right)$ and $[n-1]_{q}$ divides $[n-1]_{q_{z}}$ since $q \mid q_{z}$. Therefore, $[n]_{q}$ divides $[n-1]_{q_{y}}[n-1]_{q_{z}}$.

Next, suppose $n$ is coprime to $\operatorname{deg}(x)$ for all $x \in R$, i.e., $\wp(R, n)=1$. We treat $q$ as a parameter and write $q^{n}-1=(q-1) \Phi(q)$. The formula in Theorem 3.13 becomes

$$
\frac{(-1)^{n-1} \prod_{x \in R}[n-1]_{q_{x}}+[n-1]_{q} n^{\# R-1}(\Phi(q)-1)}{\Phi(q)[n-1]_{q}} .
$$

If $n$ is prime, then $\Phi(q)=q^{n-1}+q^{n-2}+\cdots+q+1$ is coprime to $[n-1]_{q}$ (as polynomials in $\mathbb{C}[q])$. Since $[n-1]_{q}$ obviously divides the numerator, it is enough to show that $\Phi(q)$ divides the numerator. $\Phi(q)=\left(q-\zeta_{1}\right) \cdots\left(q-\zeta_{n-1}\right)$ has degree $n-1$ 
and its zeros $\left\{\zeta_{1}, \ldots, \zeta_{n-1}\right\}$ are the primitive $n$th roots of 1 . It is enough to show that any $\zeta \in\left\{\zeta_{1}, \ldots, \zeta_{n-1}\right\}$ is a zero of the numerator. Note that $\left\{\zeta, \ldots, \zeta^{n-1}\right\}=$ $\left\{\zeta_{1}, \ldots, \zeta_{n-1}\right\}$. Let $x \in R$ and $m:=\operatorname{deg}(x)$. Consider

$$
f(q)=[n-1]_{q_{x}}=\left(q^{m(n-1)}-1\right)\left(q^{m(n-2)}\right) \cdots\left(q^{m}-1\right) .
$$

Since $m$ is coprime to $n$,

$$
\begin{aligned}
f(\zeta) & =\left(\zeta^{m(n-1)}-1\right)\left(\zeta^{m(n-2)}-1\right) \cdots\left(\zeta^{m}-1\right) \\
& =\prod_{i=1}^{n-1}\left(\zeta_{i}-1\right)=(-1)^{n-1} \Phi(1)=(-1)^{n-1} n
\end{aligned}
$$

Hence the numerator with $q=\zeta$ is equal to

$$
(-1)^{(n-1)(\# R+1)} n^{\# R}-(-1)^{n-1} n^{\# R}=0 .
$$

Remark 3.18. Let $n$ be arbitrary and $\bar{\Gamma}^{\prime}$ be a normal, finite index, torsion-free subgroup of $\bar{\Gamma}$. Since $\bar{\Gamma}^{\prime}$ is a discrete, cocompact, torsion-free subgroup of $G$, by a result of Garland [5] and Casselman [3], $H^{i}\left(\bar{\Gamma}^{\prime}, \mathbb{Q}\right) \cong H^{i}\left(\bar{\Gamma}^{\prime} \backslash \mathcal{B}, \mathbb{Q}\right)=0$ for $1 \leq i \leq n-2$. There is a spectral sequence $H^{i}\left(\bar{\Gamma} / \bar{\Gamma}^{\prime}, H^{j}\left(\bar{\Gamma}^{\prime} \backslash \mathcal{B}, \mathbb{Q}\right)\right) \Rightarrow H^{i+j}(\bar{\Gamma} \backslash \mathcal{B}, \mathbb{Q})$. Since $\bar{\Gamma} / \bar{\Gamma}^{\prime}$ is finite, $H^{i}\left(\bar{\Gamma} / \bar{\Gamma}^{\prime}, \mathbb{Q}\right)=0$ for $i \geq 1$. We conclude that

$$
H^{i}(\Gamma \backslash \mathcal{B}, \mathbb{Q})=0 \text { for } 1 \leq i \leq n-2 .
$$

Since $\operatorname{dim}_{\mathbb{Q}} H^{0}(\Gamma \backslash \mathcal{B}, \mathbb{Q})=1$,

$$
\operatorname{dim}_{\mathbb{Q}} H^{n-1}(\Gamma \backslash \mathcal{B}, \mathbb{Q})=(-1)^{n-1}(\chi(\Gamma \backslash \mathcal{B})-1) .
$$

Thus, when $n$ is prime, Theorem 3.13 provides an explicit expression for the dimension of this cohomology group. Moreover, Corollary 3.14 implies that this number is always a multiple of $q$.

Theorem 3.19. Suppose $n$ is prime, and $R=\{x, y\}$ with $\operatorname{deg}(x)=\operatorname{deg}(y)=1$. For $0 \leq i \leq n-1$, denote by $\theta_{i}$ the number of $i$-simplices in $\Gamma \backslash \mathcal{B}$. Then $\theta_{0}=n$, and for $1 \leq i \leq n-1$,

$$
\theta_{i}=\frac{n}{(i+1)} \frac{(q-1)}{\left(q^{n}-1\right)} \sum_{\substack{\mathbf{p} \in \operatorname{Par}(n) \\
\ell(\mathbf{p})=i+1}}\left[\begin{array}{l}
n \\
\mathbf{p}
\end{array}\right]_{q} .
$$

Proof. In general, by Theorem 3.13, the number of vertices in $\Gamma \backslash \mathcal{B}$ whose preimages have stabilizers isomorphic to $\mathbb{F}_{q^{n}}^{\times}$is $\wp(R, n) n^{\# R-1}$; the other $\theta_{0}-\wp(R, n) n^{\# R-1}$ vertices have preimages with stabilizers isomorphic to $\mathbb{F}_{q}^{\times}$. Moreover, $\Gamma_{v} / \mathbb{F}_{q}^{\times}$acts freely on the simplices containing $v$. Denoting $\operatorname{deg}_{\mathcal{B}}^{i}=\operatorname{deg}_{\mathcal{B}}^{i}(v)$ for a vertex $v \in \mathcal{B}$, we can express all $\theta_{i}, 1 \leq i \leq n-1$, as linear functions of $\theta_{0}$ :

$$
\theta_{i}=\frac{\operatorname{deg}_{\mathcal{B}}^{i}}{i+1}\left(\frac{(q-1) \wp(R, n) n^{\# R-1}}{q^{n}-1}+\left(\theta_{0}-\wp(R, n) n^{\# R-1}\right)\right) ;
$$

we count the number of $i$-simplices in $\Gamma \backslash \mathcal{B}$ containing a given vertex, add these numbers over all vertices, and then divide by $(i+1)$ since every $i$-simplex has exactly $(i+1)$ vertices. On the other hand, $\chi(\Gamma \backslash \mathcal{B})=\sum_{i=0}^{n-1}(-1)^{i} \theta_{i}$. If we substitute into this formula the expressions in (3.2), then we get a linear relation between $\theta_{0}$ and $\chi$. Since Lemma 2.5 gives a formula for the generalized degrees $\operatorname{deg}_{\mathcal{B}}^{i}$ and Theorem 3.13 gives a formula for $\chi(\Gamma \backslash \mathcal{B})$, one can always compute the numbers $\theta_{i}$ in each specific case. 
From the previous discussion, it is clear that to prove the theorem it is enough to show that $\theta_{0}=n$. When $R=\{x, y\}$ and $\operatorname{deg}(x)=\operatorname{deg}(y)=1$,

$$
\chi(\Gamma \backslash \mathcal{B})=\frac{(q-1)}{\left(q^{n}-1\right)}\left((-1)^{n-1}[n-1]_{q}-n\right)+n .
$$

Now, using the linear relation between $\chi(\Gamma \backslash \mathcal{B})$ and $\theta_{0}$, one easily checks that $\theta_{0}=n$ is equivalent to the identity

$$
1+\sum_{i=1}^{n-1}(-1)^{i} \frac{1}{i+1} \sum_{\substack{\mathbf{p} \in \operatorname{Par}(n) \\
\ell(\mathbf{p})=i+1}}\left[\begin{array}{l}
n \\
\mathbf{p}
\end{array}\right]_{q}=(-1)^{n-1} \frac{1}{n}[n-1]_{q},
$$

which is the statement of Lemma 2.7.

Remark 3.20. Theorem 3.19 says that in the case when $R=\{x, y\}$ and $\operatorname{deg}(x)=$ $\operatorname{deg}(y)=1$ one can visualize $\Gamma \backslash \mathcal{B}$ as

$$
\theta_{n-1}=\prod_{i=1}^{n-1} \frac{q^{i}-1}{q-1}
$$

$(n-1)$-simplices glued to each other at their vertices and along some of their higher dimensional faces. How exactly the higher dimensional faces are glued is easy to describe for small $n$, but becomes complicated as $n$ grows.

Suppose $n=2$. Then $\theta_{0}=2, \theta_{1}=1$. Hence $\Gamma \backslash \mathcal{B}$ is a segment (= two vertices joined by an edge).

Suppose $n=3$. Then $\theta_{0}=\theta_{1}=3$ and $\theta_{2}=q+1$. Hence $\Gamma \backslash \mathcal{B}$ consists of $(q+1)$ triangles glued together along their boundaries.

\section{REFERENCES}

[1] G. Andrews, The theory of partitions, Cambridge Univ. Press, 1984. MR.1634067(99c:11126)

[2] K. Brown, Buildings, Springer, 1989. MR969123 (90e:20001)

[3] W. Casselman, On a p-adic vanishing theorem of Garland, Bull. Amer. Math. Soc. 80 (1974), 1001-1004. MR0354933 (50:7410)

[4] M. Denert and J. Van Geel, The class number of hereditary orders in non-Eichler algebras over global function fields, Math. Ann. 282 (1988), 379-393. MR967020 (90d:11126)

[5] H. Garland, p-adic curvature and the cohomology of discrete subgroups of p-adic groups, Ann. of Math. (2) 97 (1973), 375-423. MR0320180 (47:8719)

[6] E.-U. Gekeler, Automorphe Formen über $\mathbb{F}_{q}(T)$ mit kleinem Führer, Abh. Math. Sem. Univ. Hamburg 55 (1985), 111-146. MR831522 (87e:11063)

[7] E.-U. Gekeler and U. Nonnengardt, Fundamental domains of some arithmetic groups over function fields, Internat. J. Math. 6 (1995), 689-708. MR1351161 (96i:11043)

[8] E.-U. Gekeler and M. Reversat, Jacobians of Drinfeld modular curves, J. Reine Angew. Math. 476 (1996), 27-93. MR1401696 (97f:11043)

[9] C. Latimer and C. MacDuffee, A correspondence between classes of ideals and classes of matrices, Ann. of Math. (2) 34 (1933), 313-316. MR.1503108

[10] G. Laumon, Cohomology of Drinfeld modular varieties: Part I, Cambridge Univ. Press, 1996. MR.1381898 (98c:11045a)

[11] J. Munkres, Elements of algebraic topology, Addison-Wesley, 1984. MR755006 (85m:55001)

[12] M. Papikian, Genus formula for modular curves of D-elliptic sheaves, Arch. Math. 92 (2009), 237-250. MR2496675 (2010a:11105)

[13] M. Papikian, Modular varieties of D-elliptic sheaves and the Weil-Deligne bound, J. Reine Angew. Math. 626 (2009), 115-134. MR2492991

[14] I. Reiner, Maximal orders, Academic Press, 1975. MR0393100 (52:13910)

[15] J.-P. Serre, Cohomologie des groupes discrets, Ann. Math. Studies, 70, Princeton University Press, 1970, 77-169. MR0385006 (52:5876) 
[16] C. Soulé, Chevalley groups over polynomial rings, London Math. Soc. Lect. Note Ser., 36, Cambridge Univ. Press, 1979, 359-367. MR564437 (81g:20080)

[17] M.-F. Vignéras, Arithmétiques des algèbres de quaternions, Lecture Notes in Math., 800, Springer-Verlag, 1980. MR.580949 (82i:12016)

Department of Mathematics, Pennsylvania State University, University Park, PennSYLVANIA 16802

E-mail address: papikian@math.psu.edu 